\title{
Original
}

\section{Nefrectomia radical laparoscópica en piezas quirúrgicas de gran tamaño}

\author{
Sergio Alonso y Gregorio, S. Sánchez, C. Soler, A. Rando, M. Girón, Ángel J. Tabernero Gómez, \\ A. Aguilera, Jesús Cisneros Ledo, Javier de la Peña Barthel
}

Servicio de Urología. Hospital Universitario La Paz. Madrid. España.

\begin{abstract}
Resumen
Introducción: La cirugía retroperitoneal constituye la indicación más indiscutible para el abordaje laparoscópico. Todavía persisten indicaciones de cirugía abierta en función de la complejidad del caso y de la experiencia del cirujano.

Objetivos: El objetivo principal es comunicar nuestra experiencia de nefrectomía radical laparoscópica en pacientes con tumores $\geq 7 \mathrm{~cm}$ o con piezas quirúrgicas cuyo peso haya sido $\geq 700 \mathrm{~g}$. Analizaremos sus características, la tasa de complicaciones intraoperatorias, el tiempo quirúrgico, el curso postoperatorio así como la estancia postoperatoria.

Material y métodos: Desde julio del 2004 hasta julio del 2008 hemos realizado un total de 104 nefrectomías radicales laparoscópicas. Hemos seleccionado un subgrupo de 41 pacientes con criterios de pieza quirúrgica de gran tamaño.

Resultados: El tiempo quirúrgico medio ha sido de 184,3 minutos. Hemos tenido un caso de reconversión a cirugía abierta y un fallecimiento intraoperatorio por causa cardiológico. La estancia media global ha sido de 3,51 días. La tasa de complicaciones y el curso postoperatorio son comparables a toda la serie.

Discusión: La nefrectomía laparoscópica es técnicamente menos compleja que la prostatectomía radical laparoscópica. Sin embargo, sus posibles complicaciones entrañan una gravedad mucho mayor. Los casos más complejos han de ser abordados por vía laparoscópica sólo cuando existe una dilatada experiencia. En general, en nuestro centro relegamos a cirugía abierta las piezas que sobrepasan la línea media y los casos con trombo tumoral que afecte a la vena renal principal y a la VCI.
\end{abstract}

Palabras clave: Laparoscopia. Nefrectomía. Cáncer renal.

\section{Laparoscopic radial nephrectomy in big surgical specimens}

\begin{abstract}
Introduction: The retroperitoneal surgery is the clearer indication for the laparoscopic approach. Still there are indications for open surgery because of the complexity of the patient and the surgeon experience.

Objectives: The main objective is to report our experience of laparoscopic radical nephrectomies in patient with tumors greater than $7 \mathrm{~cm}$. or surgical specimens with more than $700 \mathrm{~g}$ of weight. We analyze their characteristics, complications rate, surgical time, postoperative outcome and hospital stay.

Materials and methods: Since july 2004 to july 2008 we have performed 104 laparoscopic radical nephrectomies. We have selected 41 patients with characteristics of big surgical specimens.

Results: The average surgical time has been of $184,3 \mathrm{~min}$. Only 1 case has been converted into open surgery and only 1 intraoperative death because of cardiologic arrhythmia. The average hospital stay has been of 3,51 days. The complications rate and postoperative outcome are similar to the whole series.

Discussion: Laparoscopic nephrectomy is technically less complex than radical prostatectomy. However, their possible complication implies a much bigger severity. The more complex cases must be approach laparoscopically only after a wide experience. Actually, in our department we usually indicate an open approach when surgical specimen crossover the abdominal middle line and in those cases where the tumoral thrombus affect the main renal vein and/or the vena cava.
\end{abstract}

Keywords: Laparoscopy. Nephrectomy. Renal cancer.

$\mathrm{D}$ esde que en 1991 Clayman et al. describieran las primeras cirugías laparoscópicas renales $^{1,2}$, la expansión de esta técnica ha sido imparable. El desarrollo de la cirugía laparoscópica en nuestro país ha sido mucho más tardío aunque en la actualidad ya forma parte de nuestra práctica rutinaria.
La cirugía laparoscópica en general y la retroperitoneal en particular, puede desarrollarse tanto por vía transperitoneal como por vía retroperitoneal, existiendo escuelas con preferencias diferentes en base a su propia experiencia, pero hasta la fecha ninguna se ha impuesto sobre la otra $^{3-5}$. 
En el caso concreto de la nefrectomía radical laparoscópica, podemos afirmar en base a los seguimientos oncológicos publicados de 5-7 años ${ }^{6-8}$ que se constituirá como el tratamiento quirúrgico de primera línea. Sin embargo, el carcinoma renal se puede presentar en ocasiones como masas de gran tamaño, con afectación de la vena renal principal o de la vena cava inferior, con afectación local importante, conglomerados adenopáticos hiliares, etc. lo que plantea la pregunta sobre si existen límites a la cirugía laparoscópica, y si éstos son salvables con la cirugía abierta convencional.

El objetivo principal es comunicar nuestra experiencia de nefrectomía radical laparoscópica en pacientes con tumores $\geq 7 \mathrm{~cm}$ o con piezas quirúrgicas cuyo peso haya sido $\geq 700 \mathrm{~g}$. Analizaremos la tasa de complicaciones intraoperatorias, el tiempo quirúrgico, el curso postoperatorio así como la estancia postoperatoria. Igualmente, realizaremos un análisis crítico de las indicaciones de la cirugía laparoscópica en casos complejos como pueden ser el tamaño tumoral y/o el tamaño de la pieza quirúrgica.

\section{MATERIAL Y MÉTODOS}

Desde julio 2004 hasta julio del 2008 hemos realizado un total de 104 nefrectomías radicales laparoscópicas ${ }^{9}$. Todos los procedimientos han sido realizados por 2 cirujanos (S.A. y A.A). Todos los procedimientos han sido realizados mediante un acceso transperitoneal.

Hemos seleccionado un subgrupo de pacientes en función del tamaño tumoral informado por el patólogo como $\geq$ de $7 \mathrm{~cm}$ de diámetro máximo y/o peso de la pieza quirúrgica $\geq$ de 700 gr. Con estos criterios de inclusión hemos realizado un análisis retrospectivo sobre 13 pacientes con tumores de gran tamaño $(\geq 7 \mathrm{~cm}), 22$ pacientes con piezas quirúrgicas de gran tamaño ( $\geq 700 \mathrm{~g}$ ) y otros 6 pacientes que cumplen ambos requisitos. En total hemos incluido a 41 pacientes.

\section{RESULTADOS}

La edad media de los pacientes es de 63,05 años (34-85) siendo 11 mujeres y 30 varones. El tiempo quirúrgico medio ha sido de 184,3 minutos (115285) sin evidenciar diferencias entre los tres subgrupos y que es comparable al tiempo quirúrgico global del resto de la serie de nefrectomías radicales de 159,23 minutos (95-240) (Tablas 1 y 2).
Tabla 1. Comparación grupos de casos complejos

\begin{tabular}{lcc}
\hline & $\begin{array}{c}\text { Tiempo quirúrgico } \\
\text { min }\end{array}$ & Estancia \\
\hline Tamaño $\geq 7 \mathrm{~cm}$ & 188,18 & 3,69 \\
Peso $\geq 700$ gr. & 180,52 & 3,04 \\
Ambos & 189,16 & 3,66 \\
\hline
\end{tabular}

Tabla 2: Comparación grupo "casos complejos" con resto de la serie

\begin{tabular}{lcc}
\hline & Tiempo quirúrgico & Estancia \\
\hline $\begin{array}{l}\text { Grandes } \\
\text { especímenes } \\
\mathrm{n}=41\end{array}$ & 184,3 & 3,51 \\
$\begin{array}{l}\text { Resto serie } \\
\mathrm{n}=63\end{array}$ & 159,23 & 3,47 \\
\hline
\end{tabular}

Tan sólo hemos tenido un caso de reconversión a cirugía abierta por imposibilidad técnica $(2,4 \%)$ dada la progresión local de la enfermedad, ningún caso de reintervención quirúrgica, así como tan sólo hemos tenido un caso de transfusión postoperatoria y ningún caso de transfusión intraoperatoria. Hemos tenido un fallecimiento intraoperatorio en un varón de 54 años secundaria a una fibrilación ventricular y ningún caso en el postoperatorio inmediato. En el resto de la serie hemos tenido 4 reconversiones $(6,5 \%)$ a cirugía abierta ( 3 por causa vascular y 1 por progresión local), 2 reintervenciones quirúrgicas en el comienzo de la experiencia por sangrado $(3,27 \%), 1$ caso de reingreso por hematoma del lecho quirúrgico, así como 1 único caso de transfusión postoperatoria.

Como complicaciones postoperatorias destacan un paciente con angina de pecho que obligó su traslado al Servicio de Cardiología, 4 pacientes con insuficiencia renal leve-moderada transitoria, 1 paciente con broncoespasmo en el postoperatorio que se manejaron con tratamiento médico con éxito y 1 único caso de sobreinfección de la herida quirúrgica en el único paciente de los 41 de este estudio que se realizó mediante laparoscopia manoasistida. No existen diferencias reseñables con las complicaciones médicas presentadas en el resto de la serie.

La estancia media global ha sido de 3,51 días (27 días) sobre 38 casos, excluidos el fallecimiento intraoperatorio, el traslado a otro Servicio y el paciente reconvertido a cirugía abierta. Cabe destacar que 
no hay diferencia entre los grupos en términos de estancia postoperatoria ni en comparación con la estancia postoperatoria de toda la serie de 3,47 días (Tablas 1 y 2).

\section{DISCUSIÓN}

Podemos decir sin miedo a equivocarnos que no existe ninguna duda sobre los beneficios de la laparoscopia en la cirugía retroperitoneal. Tales beneficios han sido descritos en relación a la tasa de transfusión, requerimientos analgésicos en el postoperatorio, estancia postoperatoria y recuperación de la vida cotidiana, así como beneficios cosméticos ${ }^{10}$. Dados estos evidentes beneficios, la tendencia de todos los grupos es intentar ofrecer el abordaje laparoscópico al mayor número posible de procedimientos.

En general, la cirugía retroperitoneal laparoscópica implica una menor curva de aprendizaje y una menor dificultad técnica que la prostatectomía radical laparoscópica, lo que ha motivado que sean muchos los grupos que han iniciado su experiencia laparoscópica en la cirugía renal. Sin embargo, las potenciales complicaciones de la cirugía retroperitoneal pueden ser de gran gravedad e incluso condicionar un riesgo vital. Por todo ello, sólo cuando se adquiere una experiencia adecuada parece razonable abordar procedimientos de mayor complejidad como la cirugía renal conservadora y las nefrectomías de mayor complejidad.

Desde el punto de vista de la nefrectomía radical laparoscópica, existen dos características que suponen una dificultad añadida: tumores y piezas quirúrgicas de gran tamaño. Las dificultades que ofrecen estos casos pasan por el complejo manejo de tumores con posible afectación local, linfática regional y dificultades para manejar piezas de gran tamaño con el instrumental laparoscópico y las limitaciones propias de un espacio reducido ${ }^{11,12}$. Dadas estas dificultades, son varios los grupos que ante determinados casos complejos optan por la cirugía manoasistida ${ }^{13,14}$. Nuestro grupo adquirió cierta experiencia con la cirugía manoasistida al comienzo de nuestra curva de aprendizaje pero rápidamente optamos por prescindir de ella, puesto que retrasa la curva de aprendizaje aunque ofrece beneficios evidentes ante experiencias iniciales y en casos complejos con experiencias limitadas ${ }^{11-14}$. Por otro lado, creemos que con experiencias dilatadas los beneficios de la cirugía manoasistida son relativos aún ante casos de gran complejidad.
La experiencia de nuestro grupo en cirugía retroperitoneal laparoscópica se inició a finales del 2004. Un único cirujano inició la experiencia de nuestro centro, para posteriormente constituir de forma pareja a como hicimos en la prostatectomía radical laparoscópica un equipo de dos cirujanos que actualmente aglutinan toda la experiencia en nuestro centro (S.A. y A.A.). Una vez adquirida una experiencia adecuada y superada la curva de aprendizaje, decidimos abordar aquellos casos más complejos. En general, consideramos indicaciones de cirugía abierta las grandes piezas que alcancen la línea media y el trombo tumoral que afecte a la vena renal principal y/o a la vena cava inferior, aunque ya existen publicaciones sobre el abordaje laparoscópico en estos casos. Por supuesto, siempre hay casos que quedan a consideración del cirujano en función de su propia experiencia, como pueden ser masas que se proyecten sobre el hilio renal o con afectación linfática perihiliar importante, etc. o incluso los inicialmente considerados para cirugía abierta. Afortunadamente, el diagnóstico incidental de la masa renal hace que la mayoría de nuestros pacientes sean subsidiarios del abordaje laparoscópico.

En nuestra experiencia, la nefrectomía radical laparoscópica ofrece beneficios evidentes incluso en aquellos casos más complejos, como demuestran una tasa de complicaciones comparable a toda la serie de nefrectomía radical laparoscópica así como una estancia postoperatoria similar. Tan sólo existen ciertas diferencias en cuanto al tiempo quirúrgico entre ambos grupos de cuantía tan pequeña (diferencia de tiempos quirúrgicos medios de 20 minutos) que no cabe considerar otro abordaje que no sea el laparoscópico (Tabla 2).

\section{CONCLUSIONES}

La nefrectomía radial laparoscópica debe ser la indicación de primera elección para el tratamiento de cualquier masa renal. Los beneficios del abordaje laparoscópico en este campo deben obligarnos a desarrollar al máximo sus posibilidades en favor de nuestros pacientes.

Consideramos que las indicaciones de la cirugía laparoscópica renal exigen asentarse en el sentido común y la prudencia, puesto que las complicaciones intraoperatorias pueden tener consecuencias fatales. Por todo ello, sólo aquellos grupos con experiencia suficiente pueden abordar con garantías de éxito estos casos complejos como hemos puesto de manifiesto con este artículo. 


\section{REFERENCIAS}

1. Clayman RV, Kavoussi LR, Soper NJ, Dierks SM, Meretyk S, Darcy MD et al. Laparoscopic nephrectomy: initial case report J Urol. 1991 Aug;146(2):278-282.

2. Clayman RV, Kavoussi LR, Figenshau RS, Chandhoke PS, Albala DM. Laparoscopic nephroureterectomy: initial clinical case report. J Laparoendosc Surg. 1991 Dec;1(6):343-349.

3. Desai MM, Strzempkowski B, Matin SF, Steinberg AP, Ng C, Meraney AM et al. Prospective randomized comparison of transperitoneal versus retroperitoneal laparoscopic radical nephrectomy. J Urol. 2005 Jan;173(1):38-41.

4. Nambirajan T, Jeschke S, Al-Zahrani H, Vrabec G, Leeb K, Janetschek G. Prospective, randomized controlled study: transperitoneal laparoscopic versus retroperitoneoscopic radical nephrectomy. Urology. 2004 Nov;64(5):919-924.

5. Rassweiler J, Frede T, Henkel TO, Stock C, Alken P. Nephrectomy: A comparative study between the transperitoneal and retroperitoneal laparoscopic versus the open approach. Eur Urol. 1998;33(5):489-496.

6. Hemal AK, Kumar A, Gupta NP, Kumar R. Oncologic outcome of 132 cases of laparoscopic radical nephrectomy with intact specimen removal for T1-2NOMO renal cell carcinoma. World J Urol. 2007 Dec;25(6):619-626.

7. Colombo JR Jr, Haber GP, Aron M, Cocuzza M, Colombo R, Kaouk $\mathrm{J}$ et al. Oncological outcomes of laparoscopic radical nephrectomy for renal cancer. Clinics (Sao Paulo). 2007 Jun 62(3):251-256.

8. Colombo JR Jr, Haber GP, Jelovsek JE, Lane B, Novick AC, Gill IS. Seven years after laparoscopic radical nephrectomy: oncologic and renal functional outcomes. Urology. 2008 Jun;71(6): 1149-1154. Epub 2008 Mar 3.
9. Aguilera Bazán A, Alonso y Gregorio S, Cansino Alcaide R, Hidalgo Togores L, Cisneros Ledo J, de la Peña Barthel J. Retroperitoneal laparoscopic surgery; initial experience in the Hospital La Paz. Actas Urol Esp. 2005 Jul-Aug;29(7):657-661.

10. Eskicorapci SY, Teber D, Schulze M, Ates M, Stock C, Rassweiler JJ. Laparoscopic radical nephrectomy: the new gold standard surgical treatment for localized renal cell carcinoma. ScientificWorldJournal. 2007 Apr 9;7:825-836. Review.

11. Steinberg AP, Finelli A, Desai MM et al. Laparoscopic radical nephrectomy for large (greater then $7 \mathrm{~cm}$, T2) renal tumors. $\mathrm{J}$ Urol 2004 Dec;172(6 Pt 1):2172-2176.

12. Berger AD, Kanofsky JA, O`Malley RL et al. Transperitoneal laparoscopic radical nephrectomy for large (more than $7 \mathrm{~cm}$ ) renal masses. Urology 2008 Mar;71(3):421-424.

13. Stifelman MD, Handler T, Nieder AM et al. Hand-assisted laparoscopy for large renal specimens: a multi-institutional study. Urology 2003 Jan;61(1):78-82.

14. Malaeb BS, Sherwood JB, Taylor GD, Duchene DA, Broder KJ, Koeneman KS. Hand-assisted laparoscopic nephrectomy for renal masses $>9.5 \mathrm{~cm}$ : series comparison with open radical nephrectomy. Urol Oncol 2005 Sep-Oct;23(5):323-327.

Correspondencia autor: Dr. Sergio Alonso y Gregorio Servicio de Urología. Hospital Universitario La Paz Paseo de la Castellana, 261. 28046 Madrid.

Telf: 917277000

E-mail autor: garavelos@terra.es

Información artículo: Original - Laparoscopia

Trabajo recibido: marzo 2009

Trabajo aceptado: mayo 2009 